\title{
Innovation of Emergency Management Establishment of New Mode for Holographic Command and Collaborative Work
}

\author{
Liangyan JIANG \\ The second department of engineer institute in jiangsu xuzhou, Xuzhou ,Jiangsu \\ email:jshliang@sohu.com
}

\begin{abstract}
Holographic command and collaborate work are the breakthrough to traditional emergency management mode. Holographic command, as a kind of all weather, three dimensional and information-shared network interactive command platform, consists of sound, image, data and is featured with comprehensive, information-shared, real-time visible and highly efficient and intelligentized characters. With the aid of this platform, all parts involved in the significant emergency coping process can establish a more scientific and unified command and collaboration reply mechanism, in order to overcome the mass command and one-man operation issues, promote the integration of resources, optimize the work process and improve the scientificity and effectiveness in emergency management process.
\end{abstract}

Keywords-emergency management; holographic command; joint command; collaborative work

\section{Introduction}

With the accumulation of emergency management experiences in respect of natural disaster, calamity accidents and terrorist attack, people are gradually realized the importance of correct decision-making, integration of resources and collaboration work. The emergency management people are under eager help for immediate needs of overall and accurate information to make correct decisions and carry out efficient and safe rescue actions once the disaster occurred. However, due to the barriers on collection, integration and communication of information, problems of multilevel, overriding and confused orders will arise in traditional emergency management method, and thus leading to wrong decisions and weak actions. Therefore, the innovation is becoming an urgent affaire for traditional emergency management. With the rapid development of modern information network technology, the commanders are certainly capable to use advanced video, audio and data transformation techniques to realize the integration of the operation system and information platform of emergency management, build up a scientific mechanism of holographic command collaboration work and improve the scientificity and efficiency for copping the emergencies.

II. Use Modern Information Network Technology to Set up Holographic Management Network

As an all weather, three dimensional and information shared network interactive command platform, holographic command system is capable to realize the real time connection and exchange of relevant information, such as video, audio and data, between command center, onsite rescuers and emergency aid organizations. By the way of adding video and data information managing method, holographic command network is capable to overcome the barrier of incapable highly difficult rescue through single audio communication. The network is not only capable to obtain and integrate the information from the site and other channels, but also can connect multiple information channels and form the data center, which is greatly helpful to enhance the emergency command abilities.

The first one among the three elements for holographic command network is Audio. It is the primary requirement for site rescuers to speak with command center smoothly for successful emergency command. That means the main commanders, principals in all level and onsite people shall be provided with professional training and be familiar with all kinds of communication devices and techniques. General communication tools include portable tools, mobile tools and auxiliary tools. Portable tools consists of interphone, radio, cell phone, satellite phone and sound telephone; Mobile tools cover the HF radio, UHF radio, $800 \mathrm{MH}$ radio and vehicle multi band interphone; auxiliary tools include metro intercom system, building intercommunication system and coaxial-cable. What particularly mentionable is that the ACU-1000 intelligent emergency communication system equipped on site communication vehicles has powerful communication function, for it enables all kinds of radio and phone communication systems in different frequency connect with it through whole frequency range cover function and integration the devices that can't match with frequency difference in general cases. ${ }^{[1]}$

The second one is video. Site rescue will be harder if the site Internet and communication cable is broken down by earthquake, fire hazard and terrorist attack and other disasters. In such cases, one way to solve the problem is providing aerial remote sensing information by remote sensing satellite and space shuttle, the other method is providing site pictures by wireless camera or IR imaging devices. Command center will collect all these information and interlinking them with the platform and thus form a multi-functional real time image network system. The 
experts can realize the integration of video communication, site monitoring and command process by video control and command, which enables the commanders, experts and governmental authorities take part in the site rescue like they are present in the site and make visual control for site rescuers and materials and thus improve command efficiency.

The third one is data. Successful emergency command needs data support. Holographic command system consists of computer network based on emergency data management system that can be used as the data reference for risk assessment, infrastructure safety monitoring and emergency rescue. Since the fire control system of major cities consists of several subsystems, such as case accept system, automatic alarm accept system, fire control communication system, command system and information management system, a powerful and efficient database system is becoming necessary to manage all the data from fire control center. The database system shall be capable to ensure all the data of the whole system are highly consistent and complete and all the subsystems are working under smooth operation. ${ }^{[2]}$ Additionally, a kind of tiny database device, developed by researchers, has been equipped on the protecting cloth to protect the rescuer. The command center can control the position and status of rescuers through electric data tracking system once rescuers are out of connection due to sudden situation changes.

Powerful command and control functions of holographic command network are as follows:(1) Using 1080P Full HD CODEC technique to make HD information control, (2)Using route media exchange technique and distributed and graded network technology to realize real time command without any delay, (3) Using 3G networking platform to realize mobile decision making command, (4)Using integration media exchange platform of emergency command, real time image transmission, training and meetings to realize the integration of ordinary training and site working. (5) Using embedded integration wireless emergency command vehicle terminal to ensure troublefree of communication devices, (6) Using full HD TV wall and emergency control terminal to realize visual command, (7) Using intelligent command and control system to integrate equipment, materials and data together to realize centralized control,(8) Realize multi functions of intelligent command process of emergency command system, such as collaborating command, intelligent control, intelligent notice, mapping linkage and positioning system. ${ }^{[3]}$ The command system is capable to set up omni bearing communication between site, logistics, units, individual personal, decision makers and operators to improve the working efficiency under emergency cases.

\section{Set up Interconnect Holographic Network Consists of Multiple Units}

The holographic command network above mentioned is used for straight command system for single unit. There would be more parts involved in and the individual command system will change into collaborating command system when more extensive, more complex and severe emergencies occurred. Joint command is a kind of united structure that combines all emergency commanders together for efficient coordination response and their individual actions. Also, it connects the emergency command organizations together to provide a platform for making agreed decisions. ${ }^{[4]}$ Finally, it takes the concept of Network Command to form the integrated response strength by reasonably control the involved units for their close cooperation in rescue works. As a consequent, building up a wider information network system becomes necessary. Practically, America is the earliest one to set up such a multiple units interconnect holographic network. After $9 \bullet 11$ accident, America established DHS, then other federal authorities built up the emergency office and the local government also established emergency operation center. All of them are playing as command and control roles for emergency response system. To connect all these offices and centers as a national wide command network by developing a larger and information shared intelligent holographic network plays an important role for all the involved parties to take part in, directly or indirectly, the emergency rescue work through network. ${ }^{[5]}$

As an intelligent system and by using integrated management and advanced structure to combination of manufacturing platform and multimedia platform, the interconnect holographic command network completely solved the key problems of trans-department video and audio communication, all kinds of images involved and storage, transmission and isomerism of emergency data to realize the organic integration of all the information from all channels and form a standard, integrated and intelligent trans-department emergency command platform. ${ }^{[6]}$ In terms of dealing with the complex issues of emergency platform connection and integration of complex systems, including communication, computer, network and multimedia, between command center and each involved special unit, the interconnect holographic command consists of five parts: emergency base network system, emergency multimedia integration system, emergency system network security system, emergency WAN data collecting system and emergency integrated management and settlement system. Emergency base network system consists of double core loading balance and backups, convergence dual homing insert, high-performance exchange network and united intelligent management network. A stable and reliable base network can satisfy the demands of all operation systems on network resources. Emergency multimedia integration system is capable to integrate audio system, video meeting image insert from involved parts and connect department heads and experts at the first time to make quick decisions according to real site situation. Network security system is capable to provide solutions for internal and external users, such as safe interfere, long distance disaster preparation, boundary protection and internal control, to realize unified monitoring and management and ensure security of key data and timeliness of service. Emergency WAN data collecting system is capable to provide management solutions for customers by focused on BRP and integration of grid management, storage management, video management, audio management and security management. By doing this, 
all involved parts will be interconnected, intercommunicated, mutually supported and depended, each of them can timely understand the site rescue situation and make quick cooperation and solve the significant public events more efficiently.

Moreover, the interconnect holographic command system will not being limited to the number of units involved in emergency rescue works for its characters of integration and intelligence. More units take part in, more information it collects, therefore, it would be more intelligent and more efficient.

\section{Special Units Collaboration Work in Holographic Command}

The occurrence of vital emergency disaster brings a great challenge for Chinese leaders, whether from center government or local government. Leaders from all levers are responsible to take emergency response measures, all kinds of NGOs are involved in consequently. Thus, holographic command system emerged as require. The problems here are: where the command center should locate? How those all level authorities carry their responsibilities? How the units in same level corporate with each other? Although China has already established the principle of "all level are responsible and local management” in Emergency Response Law, but a scientific and reasonable emergency command and collaboration mechanism is necessarily needed for practice.

First, the character of emergency management requires the command center shall be located at the site where the emergency taken place, the chief executive of local government is the first person in charge, direct functional departments, such as police chief and fire commissioner shall be site commander and be responsible for resources integration and personal mobilization. Such a working model may shorten command chain and reduce intermediate level and enhance the command efficiency. Secondly, when the emergency is severe enough and beyond control of local government, local government may ask adjacent government for support and collaboration. If the cooperation between the local governments failed to deal with the event, they may ask help from up level government or even center government. Both or the higher level and the center government can provide resources, but neither of them shall replace the local government to manage the event. The third element is special department collaboration. Holographic command network makes it possible for different special department work together through resource integration and sharing the information. Once the emergency happened, all representatives shall quickly gather in command center and enter appointed seat and position. Meanwhile, all kinds of monitor and super big TV wall are available for commanders, decision makers and staffs can see all the rescue work and the site situation. The command center will jointly work together under unified control and according to the rescue requirement. All representatives shall carry out the decisions under the unified command of the chief commander. This is the way the holographic command system and collaboration mechanism formed and it can ensure the quick response and efficient operation of emergency command center.

\section{Conclusion}

The above mentioned concept of establishing holographic command and collaboration work mechanism in emergency management is the leapfrog development for traditional command model. This kind of new mechanism provides solutions for following problems: (1)The "information isolation" problem. By establishing an information platform that connects all units and enables them to share the information, it solved the information deviation and isomerism issue and formed a shared network command platform. (2) The possible disorder situation in emergency command. Use network resource to unify the standard for order, Use web technology to link all commanders, decision makers, government officials and rescuers together to realize unified command and ordered control and overcome the potential disorder command. (3) Individual working problem. The rescue work may suffer from segment working due to mass units and people and insufficient integration of resources. Under joint command and collaboration work mode and with unified decision, centralized command, collaborated work and information sharing mechanism, the whole efficiency of emergency management will be greatly improved.

Compare with traditional command mode, holographic command and collaboration work mechanism has the following practical features: (1) Integrity Based on using of modern network techniques and information integration technology, holographic command platform is free of the time and location limitation and it enables commanders and rescuers have an overall understanding, also, the collaboration work mechanism is helpful to carry out comprehensive management for disasters. (2) Visualized The safety and pertinence can be greatly improved due to the use of video, audio and data integration make it possible for rescuers and commanders to see almost all the rescue and command works. (3) Sharing The network interactive command platform in command center can integrate the information from all channels through intelligent process and deliver these information to all terminals, so as to ensure all level commanders can share the information and simplify the centralized decision making and collaboration work. (4) Efficiency Consists of video, audio and data information, the holographic network is capable to provide complete and correct information, make more scientific decisions and enable an effective rescue work. The collaboration work based on correct decision is capable to reduce waste and repeat work, improve the purposiveness and safety of emergency actions, and improve the performance efficiency of the decisions.

\section{References}

[1] Cheng Yue et al..Establishing A New Mode of Holographic Command and Collaborative Emergency Response. Chinese Public Administration 2011(5).

[2] Pei Jianguo. The Database Technology and Its Application in Fire Communication Command System of a City. Journal of the Chinese People's Armed Police Force Academy, 2005(10).

[3] Xue Weihu et al..The Feasibility of Buiding a Clear Visual 
Emergency Responding Command Networkr Journal of the Chinese People's Armed Police Force Academy,2008(12).

[4] Liu Tiemin. Emergency response command system and the joint command.China Public Security. Academy Edition. 2006(1).

[5] Li Chunlai et al.. The Application of Satellite Numural Audio Frequency Broadcast Technology for Information Release in
Disaster Warning. http://www.cma.gov.cn/zwgk/yingjgl/200810/ t20081022_19468.html.

[6] Hua San. Building a Standardized, Integrated and Intelligent Command Platform in Emergency Response. Informatization Construction, 2008(5). 